\title{
Static output-feedback controller design for vehicle suspensions: an effective two-step computational approach
}

\author{
Josep Rubió-Massegú1,3 Francisco Palacios-Quiñonero ${ }^{1,4}$ \\ Josep M. Rossell ${ }^{1,5}$ and Hamid Reza Karimi ${ }^{2,6}$
}

\begin{abstract}
In this paper, a novel two-step methodology is applied in designing static output-feedback controllers for a class of vehicle suspension systems. Following this approach, an effective synthesis of static output-feedback controllers can be carried out by solving two consecutive linear matrix inequality optimization problems. To illustrate the main features of the proposed design strategy, two different static output-feedback $H_{\infty}$ controllers are designed for a quarter-car suspension system. The first of those controllers uses the suspension deflection and the sprung mass velocity as feedback information, while the second one only requires the sprung mass velocity to compute the control actions. Numerical simulations indicate that, despite the restricted feedback information, the proposed static output-feedback $H_{\infty}$ controllers exhibit an excellent behavior in terms of both frequency and time responses, when compared with the corresponding state-feedback $H_{\infty}$ controller.
\end{abstract}

Keywords: vehicle suspensions; static output-feedback control; linear matrix inequalities

Published in IET Control Theory and Applications, Volume 8, Issue 15, pp. 1566 - 1574

Submitted on 8th July 2013

Accepted on 4th April 2014

Published on line on 22th July 2014

Published on 16th October 2014

"This paper is a postprint of a paper submitted to and accepted for publication in IET Control Theory and Applications and is subject to Institution of Engineering and Technology Copyright. The copy of record is available at IET Digital Library"

\footnotetext{
${ }^{1}$ Department of Applied Mathematics III, Universitat Politècnica de Catalunya (UPC), Av. Bases de Manresa 61-73, 08242-Manresa, Barcelona, Spain. ${ }^{2}$ Department of Engineering, Faculty of Engineering and Science, University of Agder (UiA), N-4898 Grimstad, Norway.

${ }^{3}$ Email: josep.rubio@upc.edu

${ }^{4}$ Email: francisco.palacios@upc.edu

${ }^{5}$ Email: josep.maria.rossell@upc.edu

${ }^{6}$ Corresponding author, email: hamid.r.karimi@uia.no
} 


\section{Introduction}

Nowadays, vehicle suspension systems have become a field of increasing relevance in control theory and applications. Taking advantage of new computing tools and efficient numerical algorithms, a significant number of advanced control strategies for active and semi-active suspension systems have been proposed to deal with control problems of growing complexity. Some interesting recent works can be found in $[1-8]$.

Information constraints and, in particular, restricted access to the state information are factors of indubitable importance $[9,10]$. When the information available for feedback purposes consists in a reduced number of linear combinations of the states, static output-feedback control strategies constitute an excellent option to facilitate a simple implementation in practice [11-15]. The design of this kind of controllers, however, leads to challenging theoretical problems and serious computational difficulties $[11,16]$. To provide a practical solution to these problems, a variety of multi-step numerical algorithms have been proposed, which allow finding suboptimal solutions with a reasonable computational cost [17-25]. These heuristic approaches typically involve a number of free parameters and, for a practical application of the method, a suitable set of parameter values has to be determined. In most cases, however, there is no satisfactory solution for this important issue, which can critically compromise the effectiveness of the method.

Recently, a new computational strategy for static outputfeedback controller design was presented in [26]. This approach considers a linear matrix inequality (LMI) formulation of the state-feedback control problem, and uses a suitable transformation of the LMI variables to obtain an LMI formulation for the static output-feedback controller. The definition of the LMI variables transformation involves a matrix $\mathbf{L}$, which can take arbitrary values. For the choice $\mathbf{L}=\mathbf{0}$, this design methodology has produced positive results in the fields of vibration control of large structures [27-30], control of offshore wind turbines [31,32] and control of active vehicle suspensions [33]. The choice $\mathbf{L}=\mathbf{0}$ has the obvious advantage of its mathematical simplicity, but it presents the drawback of ignoring the specific properties of the considered control problem.

After a detailed study of the $\mathbf{L}$ matrix properties, an improved two-step design methodology has been proposed in [34]. In the initial step, a first LMI optimization problem is solved to compute an optimal state-feedback controller. As a side product, the LMI solver provides a matrix $\mathbf{X}$ that facilitates a suitable definition of the $\mathbf{L}$ matrix. Next, the output-feedback controller is obtained by solving a second LMI optimization problem. Overall, the new approach requires solving two LMI optimization problems. Moreover, the optimal state-feedback controller computed in the first step can be used as a natural reference in the performance assessment of the static output-feedback controller.

The objective of this work is to explore the potential applicability of the new two-step design methodology in the field of vehicle suspensions. Additionally, we are also interested in providing a clear and practical presentation of the main theoretical elements of the new approach, which we believe can be of general interest for control engineers in different fields. To this end, two static output-feedback $H_{\infty}$ controllers are designed for a simplified quarter-car suspension system. The first one uses the suspension deflection and the sprung mass velocity as feedback information, while the second one only requires the sprung mass velocity to compute the control actions. The state-feedback $H_{\infty}$ controller obtained in the first step of the design procedure is used as a reference in the performance assessment. The main contribution of the paper is to provide an effective computational strategy to design static outputfeedback controllers for active vehicle suspension systems. This strategy is conceptually simple, allows taking advantage of the existing state-feedback LMI formulations, and can be implemented without determining additional parameter values.

The paper is organized as follows: Section 2 provides a minimal summary of the fundamental theoretical elements involved in the proposed two-step design methodology, and its application to the particular case of $H_{\infty}$ controller design. In Section 3, a suitable mathematical model for a quartercar suspension system is provided, and the static outputfeedback $H_{\infty}$ controllers are designed. In Section 4, a suitable set of frequency and time responses are computed to assess the effectiveness of the proposed controllers. Finally, in Section 5, some conclusions and future lines of research are briefly presented.

\section{Theoretical background}

In this section, we provide a minimal background on the design methodology for static output-feedback controllers proposed in [26] and [34]. Next, in Section 2.2 , we detail how these general ideas can be applied to the particular case of $H_{\infty}$ controller design. 


\subsection{Static output-feedback controller design}

Let us consider a state-feedback controller

$$
\mathbf{u}(t)=\mathbf{G}_{s} \mathbf{x}(t),
$$

where $\mathbf{u}(t) \in \mathbb{R}^{m}$ and $\mathbf{x}(t) \in \mathbb{R}^{n}$ denote the control and state vectors, respectively, and $\mathbf{G}_{s} \in \mathbb{R}^{m \times n}$ is the state gain matrix. Let us also assume that this state-feedback controller can be designed by solving an LMI optimization problem of the form

$$
\left\{\begin{array}{l}
\operatorname{minimize} h(\mathbf{X}, \mathbf{Y}, \boldsymbol{\zeta}) \\
\text { subject to } F(\mathbf{X}, \mathbf{Y}, \boldsymbol{\zeta})<\mathbf{0}, \mathbf{X}>\mathbf{0}
\end{array}\right.
$$

where $h$ and $F$ are given affine maps, $\mathbf{X} \in \mathbb{R}^{n \times n}$ and $\mathbf{Y} \in$ $\mathbb{R}^{m \times n}$ are variable matrices, and $\zeta \in \mathbb{R}^{p \times 1}$ is a vector that collects other LMI variables not contained in $\mathbf{X}$ and $\mathbf{Y}$. More precisely, if an optimal solution to the optimization problem (2) is attained for the triplet $\left(\tilde{\mathbf{X}}_{s}, \tilde{\mathbf{Y}}_{s}, \tilde{\boldsymbol{\zeta}}_{s}\right)$, then the state gain matrix can be computed as

$$
\mathbf{G}_{s}=\tilde{\mathbf{Y}}_{s} \tilde{\mathbf{X}}_{s}^{-1}
$$

Now, let us suppose that the available information for feedback purposes consists in a vector of observed outputs $\mathbf{y}(t) \in \mathbb{R}^{q}$, which can be written in the form

$$
\mathbf{y}(t)=\mathbf{C}_{y} \mathbf{x}(t),
$$

where $\mathbf{C}_{y}$ is an $q \times n$ matrix with full row-rank $q<n$. An interesting option in this second scenario consists in considering a static output-feedback controller

$$
\mathbf{u}(t)=\mathbf{K y}(t),
$$

which computes the control actions from the observedoutput information by means of an output gain matrix $\mathbf{K} \in$ $\mathbb{R}^{m \times q}$.

The problem of obtaining a static output-feedback controller (5) can be seen as a constrained state-feedback control problem, where the state gain matrix $\mathbf{G}$ must admit the factorization

$$
\mathbf{G}=\mathbf{K C}_{y} .
$$

When an LMI formulation of the form (2) is available for the state-feedback controller design, the static outputfeedback controller (5) can be computed by solving the following optimization problem:

$$
\left\{\begin{array}{l}
\operatorname{minimize} h(\mathbf{X}, \mathbf{Y}, \boldsymbol{\zeta}) \\
\text { subject to } F(\mathbf{X}, \mathbf{Y}, \boldsymbol{\zeta})<\mathbf{0}, \mathbf{X}>\mathbf{0},(\mathbf{X}, \mathbf{Y}) \in \mathscr{M}
\end{array}\right.
$$

where $\mathscr{M}$ is the set of all pairs of matrices $(\mathbf{X}, \mathbf{Y})$ for which there exists an $m \times q$ matrix $\mathbf{K}$ satisfying the matrix equation

$$
\mathbf{Y X}^{-1}=\mathbf{K C}_{y} .
$$

Using the results presented in [26], an effective computational strategy to deal with the non-convex optimization problem (7) and computing the output gain matrix $\mathbf{K}$ can be defined as follows:

(S1) Choose a suitable $(n-q) \times q$ matrix $\mathbf{L}$ and compute

$$
\mathbf{R}=\mathbf{C}_{y}^{\dagger}+\mathbf{Q} \mathbf{L},
$$

where $\mathbf{Q}$ is an $n \times(n-q)$ matrix whose columns are a basis of the nullspace of $\mathbf{C}_{y}$, and $\mathbf{C}_{y}^{\dagger}=\mathbf{C}_{y}^{T}\left(\mathbf{C}_{y} \mathbf{C}_{y}^{T}\right)^{-1}$ is the Moore-Penrose pseudoinverse of $\mathbf{C}_{y}$.

(S2) Solve the following LMI optimization problem with variables $\mathbf{X}_{Q}, \mathbf{X}_{R}, \mathbf{Y}_{R}$ and $\zeta$ :

$$
\left\{\begin{array}{l}
\operatorname{minimize} \hat{h}\left(\mathbf{X}_{Q}, \mathbf{X}_{R}, \mathbf{Y}_{R}, \boldsymbol{\zeta}\right) \\
\text { subject to } \hat{F}\left(\mathbf{X}_{Q}, \mathbf{X}_{R}, \mathbf{Y}_{R}, \boldsymbol{\zeta}\right)<\mathbf{0}, \mathbf{X}_{Q}>\mathbf{0}, \mathbf{X}_{R}>\mathbf{0}
\end{array}\right.
$$

where $\mathbf{X}_{Q} \in \mathbb{R}^{(n-q) \times(n-q)}$ and $\mathbf{X}_{R} \in \mathbb{R}^{q \times q}$ are symmetric positive-definite matrices, $\mathbf{Y}_{R} \in \mathbb{R}^{m \times q}$ and $\zeta \in$ $\mathbb{R}^{p \times 1}$ are arbitrary matrices, and the functions $\hat{h}$ and $\hat{F}$ are defined as

$$
\begin{aligned}
& \hat{h}\left(\mathbf{X}_{Q}, \mathbf{X}_{R}, \mathbf{Y}_{R}, \boldsymbol{\zeta}\right)=h\left(\mathbf{Q} \mathbf{X}_{Q} \mathbf{Q}^{T}+\mathbf{R} \mathbf{X}_{R} \mathbf{R}^{T}, \mathbf{Y}_{R} \mathbf{R}^{T}, \boldsymbol{\zeta}\right) \\
& \hat{F}\left(\mathbf{X}_{Q}, \mathbf{X}_{R}, \mathbf{Y}_{R}, \boldsymbol{\zeta}\right)=F\left(\mathbf{Q} \mathbf{X}_{Q} \mathbf{Q}^{T}+\mathbf{R} \mathbf{X}_{R} \mathbf{R}^{T}, \mathbf{Y}_{R} \mathbf{R}^{T}, \boldsymbol{\zeta}\right)
\end{aligned}
$$

If an optimal solution to the LMI optimization problem (10) is obtained for the quartet $\left(\tilde{\mathbf{X}}_{Q}, \tilde{\mathbf{X}}_{R}, \tilde{\mathbf{Y}}_{R}, \tilde{\boldsymbol{\zeta}}\right)$, then the triplet $(\tilde{\mathbf{X}}, \tilde{\mathbf{Y}}, \tilde{\boldsymbol{\zeta}})$ with

$$
\tilde{\mathbf{X}}=\mathbf{Q} \tilde{\mathbf{X}}_{Q} \mathbf{Q}^{T}+\mathbf{R} \tilde{\mathbf{X}}_{R} \mathbf{R}^{T}, \quad \tilde{\mathbf{Y}}=\tilde{\mathbf{Y}}_{R} \mathbf{R}^{T},
$$

defines a feasible solution of the optimization problem (7), and the matrix equation (8) is satisfied by $\tilde{\mathbf{X}}, \tilde{\mathbf{Y}}$ and the output gain matrix

$$
\mathbf{K}=\tilde{\mathbf{Y}}_{R} \tilde{\mathbf{X}}_{R}^{-1} .
$$

To date, this computational procedure has been successfully applied to design static output-feedback controllers in the fields of vibration control of large structures [27-30], control of offshore wind turbines [31,32] and control of active vehicle suspensions [33]. In all these works, a zero matrix $\mathbf{L}$ was selected in the step (S1). The choice $\mathbf{L}=\mathbf{0}$ leads to $\mathbf{R}=\mathbf{C}_{y}^{\dagger}$ in (9), and has the obvious advantage of its mathematical simplicity. This option, however, presents 
the drawback of ignoring the specific properties of the considered control problem.

While developing the aforementioned applications, it became apparent that a suitable choice of the matrix $\mathbf{L}$ can exert a critical influence on both, the feasibility of the optimization problem (10), and the optimality level of the feasible triplet $(\tilde{\mathbf{X}}, \tilde{\mathbf{Y}}, \tilde{\boldsymbol{\zeta}})$. A detailed study of some relevant properties of the matrix $\mathbf{L}$ has been recently presented in [34]. The results obtained in that work led the authors to propose the following $\mathbf{L}$-matrix for the step (S1):

$$
\mathbf{L}=\mathbf{Q}^{\dagger} \tilde{\mathbf{X}}_{s} \mathbf{C}_{y}^{T}\left(\mathbf{C}_{y} \tilde{\mathbf{X}}_{s} \mathbf{C}_{y}^{T}\right)^{-1},
$$

where $\mathbf{Q}^{\dagger}=\left(\mathbf{Q}^{T} \mathbf{Q}\right)^{-1} \mathbf{Q}^{T}$ denotes the Moore-Penrose pseudoinverse of $\mathbf{Q}$, and $\tilde{\mathbf{X}}_{s}$ is the $\mathbf{X}$-matrix corresponding to an optimal solution $\left(\tilde{\mathbf{X}}_{s}, \tilde{\mathbf{Y}}_{s}, \tilde{\boldsymbol{\zeta}}_{s}\right)$ of the LMI optimization problem (2) associated to the state-feedback controller design. This choice of the matrix $\mathbf{L}$ has proved to be particularly effective in the field of seismic protection of large structures, and it will be used in the present paper to obtain static output-feedback controllers for a quarter-car suspension system with satisfactory results.

\section{$2.2 H_{\infty}$ controllers}

Let us consider a system of the form

$$
\left\{\begin{array}{l}
\dot{\mathbf{x}}(t)=\mathbf{A x}(t)+\mathbf{B u}(t)+\mathbf{B}_{w} \mathbf{w}(t), \\
\mathbf{z}(t)=\mathbf{C} \mathbf{x}(t)+\mathbf{D u}(t)
\end{array}\right.
$$

where $\mathbf{x}(t) \in \mathbb{R}^{n}$ is the state vector, $\mathbf{u}(t) \in \mathbb{R}^{m}$ is the control input, $\mathbf{w}(t) \in \mathbb{R}^{r}$ is the disturbance input, $\mathbf{z}(t) \in \mathbb{R}^{d}$ is the controlled output, and $\mathbf{A}, \mathbf{B}, \mathbf{B}_{w}, \mathbf{C}$, and $\mathbf{D}$ are real constant matrices with appropriate dimensions. For a given statefeedback controller $\mathbf{u}(t)=\mathbf{G x}(t)$, the following closed-loop system results:

$$
\left\{\begin{array}{l}
\dot{\mathbf{x}}(t)=\mathbf{A}_{G} \mathbf{x}(t)+\mathbf{B}_{w} \mathbf{w}(t) \\
\mathbf{z}(t)=\mathbf{C}_{G} \mathbf{x}(t)
\end{array}\right.
$$

where

$$
\mathbf{A}_{G}=\mathbf{A}+\mathbf{B G}, \quad \mathbf{C}_{G}=\mathbf{C}+\mathbf{D G} .
$$

In the $H_{\infty}$ approach, the objective is to obtain a state gain matrix $\mathbf{G}$ that produces an asymptotically stable matrix $\mathbf{A}_{G}$ and, simultaneously, minimizes the $H_{\infty}$ norm

$$
\gamma_{G}=\left\|T_{G}\right\|_{\infty}=\sup _{\omega \in \mathbb{R}} \sigma_{\max }\left[T_{G}(\mathrm{j} \omega)\right]
$$

where $\sigma_{\max }[\cdot]$ denotes the maximum singular value and

$$
T_{G}(s)=\mathbf{C}_{G}\left(s \mathbf{I}-\mathbf{A}_{G}\right)^{-1} \mathbf{B}_{w}
$$

is the transfer function from the disturbance input to the controlled output.

According to the Bounded Real Lemma [35], for a given $\gamma>0$, the closed-loop state matrix $\mathbf{A}_{G}$ is asymptotically stable and $\gamma_{G}<\gamma$ if and only if there exists a symmetric positive-definite matrix $\mathbf{X} \in \mathbb{R}^{n \times n}$ that satisfies the matrix inequality

$$
\left[\begin{array}{cc}
\mathbf{A}_{G} \mathbf{X}+\mathbf{X} \mathbf{A}_{G}^{T}+\gamma^{-2} \mathbf{B}_{w} \mathbf{B}_{w}^{T} & * \\
\mathbf{C}_{G} X & -\mathbf{I}
\end{array}\right]<\mathbf{0}
$$

where $(*)$ denotes the transpose of the symmetric entry. Using the values of the closed-loop matrices in (17), and by introducing the new variables $\eta=\gamma^{-2}$ and $\mathbf{Y}=\mathbf{G X}$, we obtain the following LMI:

$$
\left[\begin{array}{cc}
\mathbf{A X}+\mathbf{X} \mathbf{A}^{T}+\mathbf{B Y}+\mathbf{Y}^{T} \mathbf{B}^{T}+\eta \mathbf{B}_{w} \mathbf{B}_{w}^{T} & * \\
\mathbf{C X}+\mathbf{D Y} & -\mathbf{I}
\end{array}\right]<\mathbf{0} .
$$

Hence, an optimal state-feedback $H_{\infty}$ controller can be computed by solving the LMI optimization problem

$$
\left\{\begin{array}{l}
\text { maximize } \eta \\
\text { subject to } \operatorname{LMI}(21), \quad \eta>0, \mathbf{X}>\mathbf{0} .
\end{array}\right.
$$

If the triplet $\left(\tilde{\mathbf{X}}_{s}, \tilde{\mathbf{Y}}_{s}, \tilde{\eta}_{s}\right)$ provides an optimal solution to (22), then the state gain matrix $\mathbf{G}_{s}=\tilde{\mathbf{Y}}_{s} \tilde{\mathbf{X}}_{s}^{-1}$ defines a statefeedback controller with optimal $H_{\infty}$-norm

$$
\gamma_{G_{s}}=\tilde{\eta}_{s}^{-\frac{1}{2}} .
$$

By setting $\zeta=\eta, h(\mathbf{X}, \mathbf{Y}, \eta)=-\eta$, and taking the affine $\operatorname{map} F(\mathbf{X}, \mathbf{Y}, \eta)$ as

$$
\left[\begin{array}{ccc}
\mathbf{A X}+\mathbf{X} \mathbf{A}^{T}+\mathbf{B Y}+\mathbf{Y}^{T} \mathbf{B}^{T}+\eta \mathbf{B}_{w} \mathbf{B}_{w}^{T} & * & * \\
\mathbf{C X}+\mathbf{D Y} & -\mathbf{I} & * \\
\mathbf{0} & \mathbf{0} & -\eta
\end{array}\right]
$$

the LMI optimization problem (22) can be written in the standard form presented in (2). Consequently, for a given observed output $\mathbf{y}(t)=\mathbf{C}_{y} \mathbf{x}(t)$, the general design methodology presented in Section 2.1 can be applied to obtain a static output-feedback $H_{\infty}$ controller $\mathbf{u}(t)=\mathbf{K y}(t)$ for the system (15). In this case, the LMI optimization problem (10) can be formulated as follows:

$$
\left\{\begin{array}{l}
\text { maximize } \eta \\
\text { subject to } \hat{F}\left(\mathbf{X}_{Q}, \mathbf{X}_{R}, \mathbf{Y}_{R}, \eta\right)<\mathbf{0}, \mathbf{X}_{Q}>\mathbf{0}, \mathbf{X}_{R}>\mathbf{0}
\end{array}\right.
$$

where $\hat{F}\left(\mathbf{X}_{Q}, \mathbf{X}_{R}, \mathbf{Y}_{R}, \eta\right)$ has the form given in Fig. 1. If an optimal solution to the optimization problem (25) is attained for the quartet $\left(\tilde{\mathbf{X}}_{Q}, \tilde{\mathbf{X}}_{R}, \tilde{\mathbf{Y}}_{R}, \tilde{\eta}\right)$, then the output gain 


$$
\hat{F}\left(\mathbf{X}_{Q}, \mathbf{X}_{R}, \mathbf{Y}_{R}, \eta\right)=\left[\begin{array}{ccc}
\mathbf{A Q \mathbf { Q }} \mathbf{X}_{Q} \mathbf{Q}^{T}+\mathbf{Q} \mathbf{X}_{Q} \mathbf{Q}^{T} \mathbf{A}^{T}+\mathbf{A} \mathbf{R} \mathbf{X}_{R} \mathbf{R}^{T}+\mathbf{R X}_{R} \mathbf{R}^{T} \mathbf{A}^{T}+\mathbf{B} \mathbf{Y}_{R} \mathbf{R}^{T}+\mathbf{R} \mathbf{Y}_{R}^{T} \mathbf{B}^{T}+\eta \mathbf{B}_{w} \mathbf{B}_{w}^{T} & * & * \\
\mathbf{C Q X}_{Q} \mathbf{Q}^{T}+\mathbf{C R X}_{R} \mathbf{R}^{T}+\mathbf{D} \mathbf{Y}_{R} \mathbf{R}^{T} & -\mathbf{I} & * \\
\mathbf{0} & \mathbf{0} & -\eta
\end{array}\right]
$$

Figure 1: Explicit form of the matrix $\hat{F}\left(\mathbf{X}_{Q}, \mathbf{X}_{R}, \mathbf{Y}_{R}, \eta\right)$ in the static output-feedback $H_{\infty}$ controller design

matrix $\mathbf{K}=\tilde{\mathbf{Y}}_{R} \tilde{\mathbf{X}}_{R}^{-1}$ defines an output-feedback controller $\mathbf{u}(t)=\mathbf{K y}(t)$ with an asymptotically stable closed-loop matrix $\mathbf{A}_{G_{K}}$ and an $H_{\infty}$-norm $\gamma_{G_{K}}$ that satisfies

$$
\gamma_{G_{K}} \leq \tilde{\eta}^{-\frac{1}{2}}
$$

where $\mathbf{G}_{K}=\mathbf{K C}_{y}$ is the state gain matrix associated to the output gain matrix $\mathbf{K}$.

Remark 1 It should be noted that the LMI optimization problem (25) only provides an upper bound for $\gamma_{G_{K}}$. The actual value of the $H_{\infty}$-norm corresponding to the outputfeedback controller $\mathbf{u}(t)=\mathbf{K} \mathbf{y}(t)$ can be obtained by setting $\mathbf{G}=\mathbf{G}_{K}$ in (20), and solving the LMI optimization problem

$$
\left\{\begin{array}{l}
\text { maximize } \eta \\
\text { subject to } \operatorname{LMI}(20), \quad \eta>0, \quad \mathbf{X}>\mathbf{0} .
\end{array}\right.
$$

If an optimal value $\hat{\eta}$ is obtained in (27), then we have $\gamma_{G_{K}}=\hat{\eta}^{-1 / 2}$. Alternatively, $\gamma_{G_{K}}$ can also be computed by maximizing the maximum singular value of the transfer function $T_{G_{K}}$, as indicated in (18).

\section{Application to vehicle suspensions}

In this section, the design methodology presented in Section 2 is applied to compute two different static outputfeedback $H_{\infty}$ controllers for a quarter-car suspension system. The first controller uses the suspension deflection and the sprung mass velocity as feedback information. The second controller only uses the sprung mass velocity to compute the control actions. A state-feedback $H_{\infty}$ controller is also designed, which is taken as a natural reference in the performance assessment of the proposed output-feedback controllers, and provides the matrix $\tilde{\mathbf{X}}_{s}$ to compute the $\mathbf{L}$ matrix defined in (14). The LMI optimization problems corresponding to the different controller designs have been solved with the MATLAB Robust Control Toolbox [36].

\subsection{Quarter-car suspension model}

Let us consider a lumped-mass model of a quarter-car suspension system shown in Fig. 2, where $m_{s}$ and $m_{u}$ repre-

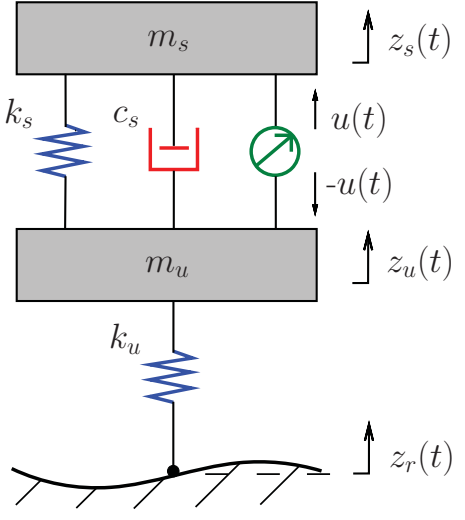

Figure 2: Quarter-car suspension model with active suspension

sent the sprung and unsprung masses, respectively; $c_{s}$ is the damping of the suspension system; $k_{s}$ and $k_{u}$ are, respectively, the suspension stiffness and the tyre stiffness; $z_{r}(t)$ is the vertical road displacement; $z_{s}(t)$ and $z_{u}(t)$ represent the vertical displacements of the sprung and unsprung masses, respectively; and $u(t)$ is the active input of the suspension system. The quarter-car motion is governed by the system of second-order differential equations

$$
\begin{aligned}
m_{s} \ddot{z}_{s}(t)= & -c_{s}\left[\dot{z}_{s}(t)-\dot{z}_{u}(t)\right]-k_{s}\left[z_{s}(t)-z_{u}(t)\right] \\
& +u(t) \\
m_{u} \ddot{z}_{u}(t)= & c_{s}\left[\dot{z}_{s}(t)-\dot{z}_{u}(t)\right]+k_{s}\left[z_{s}(t)-z_{u}(t)\right] \\
& -k_{u}\left[z_{u}(t)-z_{r}(t)\right]-u(t)
\end{aligned}
$$

which can be converted into the state-space model

$$
\dot{\mathbf{x}}(t)=\mathbf{A x}(t)+\mathbf{B} u(t)+\mathbf{B}_{w} w(t)
$$

where

$$
\mathbf{x}(t)=\left[z_{s}(t)-z_{u}(t), z_{u}(t)-z_{r}(t), \dot{z}_{s}(t), \dot{z}_{u}(t)\right]^{T}
$$

is the state vector, $w(t)=\dot{z}_{r}(t)$ is the road displacement velocity, $u(t)$ is the control input, and the matrices $\mathbf{A}, \mathbf{B}$ and 
$\mathbf{B}_{w}$ have the following form:

$$
\begin{gathered}
\mathbf{A}=\left[\begin{array}{cccc}
0 & 0 & 1 & -1 \\
0 & 0 & 0 & 1 \\
-k_{s} / m_{s} & 0 & -c_{s} / m_{s} & c_{s} / m_{s} \\
k_{s} / m_{u} & -k_{u} / m_{u} & c_{s} / m_{u} & -c_{s} / m_{u}
\end{array}\right], \\
\mathbf{B}=\left[\begin{array}{c}
0 \\
0 \\
1 / m_{s} \\
-1 / m_{u}
\end{array}\right], \mathbf{B}_{w}=\left[\begin{array}{r}
0 \\
-1 \\
0 \\
0
\end{array}\right]
\end{gathered}
$$

The following particular values of the parameters [19,37]

$$
\begin{gathered}
m_{s}=504.5 \mathrm{~kg}, m_{u}=62 \mathrm{~kg}, k_{s}=13100 \mathrm{~N} / \mathrm{m}, \\
k_{u}=252000 \mathrm{~N} / \mathrm{m}, c_{s}=400 \mathrm{Ns} / \mathrm{m},
\end{gathered}
$$

are used in the controllers design of Section 3.2 and in the numerical simulations conducted in Section 4.

The vertical body acceleration is widely used to quantify the ride comfort. Hence, a natural point of interest in the controllers design consists in minimizing the sprung mass acceleration $\ddot{z}_{S}(t)$, especially in the sensitive frequency range of 0-65 rad/s [38]. Additionally, in order to respect the suspension stroke limits and to improve the road holding ability, we are also interested in reducing the suspension deflection $z_{s}(t)-z_{u}(t)$ and the tire deflection $z_{u}(t)-z_{r}(t)$. Obviously, avoiding high levels of control effort is also desirable. Accordingly, the following vector of controlled outputs is selected:

$$
\mathbf{z}(t)=\left[\ddot{z}_{s}(t), \alpha\left(z_{s}(t)-z_{u}(t)\right), \beta\left(z_{u}(t)-z_{r}(t)\right), \rho u(t)\right]^{T},
$$

where $\alpha, \beta$ and $\rho$ are weighting coefficients that manage the tradeoff between the conflicting design requirements. Considering the first equation in (28), this vector of controlled outputs can be written in the form

$$
\mathbf{z}(t)=\mathbf{C x}(t)+\mathbf{D} u(t),
$$

with

$$
\mathbf{C}=\left[\begin{array}{cccc}
-k_{s} / m_{s} & 0 & -c_{s} / m_{s} & c_{s} / m_{s} \\
\alpha & 0 & 0 & 0 \\
0 & \beta & 0 & 0 \\
0 & 0 & 0 & 0
\end{array}\right]
$$

and

$$
\mathbf{D}=\left[\begin{array}{c}
1 / m_{s} \\
0 \\
0 \\
\rho
\end{array}\right]
$$

The following particular values of the weighting coefficients:

$$
\alpha=0.1, \quad \beta=0.2, \quad \rho=0.1 \times 10^{-3}
$$

are used to compute the controllers presented in the next section.

\subsection{Controllers design}

Following the discussion in Section 2, we begin by designing an optimal state-feedback $H_{\infty}$ controller

$$
\mathbf{u}(t)=\mathbf{G}_{s} \mathbf{x}(t),
$$

which uses the full state $\mathbf{x}(t)$ defined in (30) as feedback information. By solving the LMI optimization problem (22) with the matrices $\mathbf{A}, \mathbf{B}, \mathbf{B}_{w}, \mathbf{C}$ and $\mathbf{D}$ given by (31), (32), (36) and (37), the particular parameter values in (33) and the weighting coefficients in (38), we obtain the state gain matrix

$$
\mathbf{G}_{s}=10^{4} \times\left[\begin{array}{llll}
1.1810 & 0.2333 & -0.1096 & 0.0109
\end{array}\right],
$$

and the optimal $H_{\infty}$-norm

$$
\gamma_{G_{s}}=7.8365
$$

We also obtain the $\mathbf{X}$-matrix corresponding to this optimal solution

$$
\tilde{\mathbf{X}}_{s}=\left[\begin{array}{cccc}
0.5965 & -0.0020 & -0.5075 & -0.0085 \\
-0.0020 & 0.0020 & 0.0020 & -0.0081 \\
-0.5075 & 0.0020 & 0.7659 & -0.0015 \\
-0.0085 & -0.0081 & -0.0015 & 8.0713
\end{array}\right] \text {. }
$$

Next, we compute a first static output-feedback controller

$$
\mathbf{u}(t)=\mathbf{K}_{\mathrm{I}} \mathbf{y}_{\mathrm{I}}(t),
$$

which uses the suspension deflection and the sprung mass velocity as feedback information [19]. In this case, the observed output is given by

$$
\mathbf{y}_{\mathrm{I}}(t)=\left[z_{s}(t)-z_{u}(t), \dot{z}_{s}(t)\right]^{T},
$$

which can be written as

$$
\mathbf{y}_{\mathrm{I}}(t)=\left(\mathbf{C}_{y}\right)_{\mathrm{I}} \mathbf{x}(t)
$$

with

$$
\left(\mathbf{C}_{y}\right)_{\mathrm{I}}=\left[\begin{array}{llll}
1 & 0 & 0 & 0 \\
0 & 0 & 1 & 0
\end{array}\right]
$$


By computing the nullspace of $\left(\mathbf{C}_{y}\right)_{\mathrm{I}}$, we obtain the matrix

$$
\mathbf{Q}_{\mathrm{I}}=\left[\begin{array}{ll}
0 & 0 \\
1 & 0 \\
0 & 0 \\
0 & 1
\end{array}\right]
$$

Using the matrices (42), (46) and (47) in (14), we get

$$
\mathbf{L}_{\mathrm{I}}=\left[\begin{array}{rr}
-0.0025 & 0.0009 \\
-0.0364 & -0.0261
\end{array}\right]
$$

and, by substituting the matrices (46), (47) and (48) in (9), we finally obtain

$$
\mathbf{R}_{\mathrm{I}}=\left[\begin{array}{cc}
1 & 0 \\
-0.0025 & 0.0009 \\
0 & 1 \\
-0.0364 & -0.0261
\end{array}\right]
$$

Now, we solve the LMI optimization problem (25) with the same matrices A, B, $\mathbf{B}_{w}, \mathbf{C}$ and $\mathbf{D}$ used in the state-feedback controller design, and the matrices $\mathbf{Q}_{\mathrm{I}}$ and $\mathbf{R}_{\mathrm{I}}$ given in (47) and (49). As a result, we get the output gain matrix

$$
\mathbf{K}_{\mathrm{I}}=10^{4} \times[1.0824-0.2071]
$$

The state gain matrix $\mathbf{G}_{\mathrm{I}}=\mathbf{K}_{\mathrm{I}}\left(\mathbf{C}_{y}\right)_{\mathrm{I}}$ associated to the output gain matrix $\mathbf{K}_{\mathrm{I}}$ is given by

$$
\mathbf{G}_{\mathrm{I}}=10^{4} \times\left[\begin{array}{llll}
1.0824 & 0 & -0.2071 & 0
\end{array}\right]
$$

Setting $\mathbf{G}=\mathbf{G}_{\mathrm{I}}$ in (20), and solving the optimization problem (27), we obtain an $H_{\infty}$-norm of

$$
\gamma_{G_{I}}=7.8432
$$

To illustrate the flexibility of the proposed design methodology, we compute a second static output-feedback controller

$$
\mathbf{u}(t)=\mathbf{K}_{\mathrm{II}} \mathbf{y}_{\mathrm{II}}(t),
$$

which only uses the sprung mass velocity $\dot{z}_{s}(t)$ as feedback information. In this second case, the observed output can be written as

$$
\mathbf{y}_{\mathrm{II}}(t)=\left(\mathbf{C}_{y}\right)_{\mathrm{II}} \mathbf{x}(t)
$$

with

$$
\left(\mathbf{C}_{y}\right)_{\mathrm{II}}=\left[\begin{array}{llll}
0 & 0 & 1 & 0
\end{array}\right],
$$

and the matrices $\mathbf{L}, \mathbf{Q}$ and $\mathbf{R}$ take the following values:

$$
\mathbf{L}_{\mathrm{II}}=\left[\begin{array}{lll}
0.0026 & -0.6627 & -0.0019
\end{array}\right]^{T}
$$

$$
\mathbf{Q}_{\mathrm{II}}=\left[\begin{array}{lll}
0 & 1 & 0 \\
1 & 0 & 0 \\
0 & 0 & 0 \\
0 & 0 & 1
\end{array}\right], \quad \mathbf{R}_{\mathrm{II}}=\left[\begin{array}{r}
-0.6627 \\
0.0026 \\
1.0000 \\
-0.0019
\end{array}\right]
$$

Solving the LMI optimization problem (25) with the new values of the matrices $\mathbf{Q}$ and $\mathbf{R}$, we obtain the output gain

$$
\mathbf{K}_{\mathrm{II}}=-8.9703 \times 10^{3}
$$

which has an associated state gain matrix

$$
\mathbf{G}_{\mathrm{II}}=\mathbf{K}_{\mathrm{II}}\left(\mathbf{C}_{y}\right)_{\mathrm{II}}=10^{3} \times\left[\begin{array}{llll}
0 & 0 & -8.9703 & 0
\end{array}\right],
$$

and $H_{\infty}$-norm

$$
\gamma_{G_{\mathrm{II}}}=7.9068
$$

Remark 2 In the first step of the proposed design procedure, the objective is to find a suitable state-feedback controller. This ideal controller has full access to the state information and must satisfy the performance requirements of the problem under consideration. Clearly, if no satisfactory solution can be found for this exploratory step, the possibility of obtaining a suitable static output-feedback controller should be reconsidered. The output-feedback controller presented in [19] has been taken as a reference to compute the control gain matrix $\mathbf{G}_{s}$ given in (40). In what follows, we will assume that $\mathbf{G}_{s}$ defines a suitable statefeedback controller for the active suspension system introduced in Section 3.1.

Remark 3 Note that all the controllers presented in this section have been computed using the same controlled output $\mathbf{z}(t)$ defined in (35)-(38). For this choice of $\mathbf{z}(t)$, the state-feedback controller defined by $\mathbf{G}_{s}$ attains the optimal $H_{\infty}$-norm $\gamma_{G_{s}}=7.8365$. A suboptimal $\gamma$-value will be produced by any static output-feedback controller designed using the same $\mathbf{z}(t)$. Looking at the $\gamma$-value in (52), it can be seen that the output-feedback controller (43) is practically optimal. Comparing the $\gamma$-values in (41) and (60), it can also be appreciated that the $H_{\infty}$-norm achieved by the second output-feedback controller (53) exceeds the optimal $\gamma$-value (41) in less than a $1 \%$. From a practical point of view, the behavior of these almost-optimal controllers is often very similar to the behavior exhibited by the optimal state-feedback controller. The numerical simulations carried out in Section 4 illustrate this fact.

Remark 4 An output-feedback controller design for a quarter-car suspension model using a single-step procedure can be found in [33]. In this preliminary work, the state 
variables are $z_{s}(t), z_{u}(t), \dot{z}_{s}(t), \dot{z}_{u}(t)$, and a static outputfeedback controller of the form given in (43) is computed by using $\mathbf{R}=\mathbf{C}_{y}^{\dagger}$, which can be considered as a particular case of (9) with $\mathbf{L}=\mathbf{0}$. However, in this case, the corresponding LMI is unfeasible for an output-feedback controller of the form (53). For the state variables (30), proposed in [19] and used in the present paper, the attempt of computing the output-feedback controllers (43) and (53) by using a null matrix $\mathbf{L}$ in (9) also fails, and the corresponding LMI optimization problems are reported to be infeasible by the MATLAB LMI solver. Similar feasibility problems associated to the choice $\mathbf{L}=\mathbf{0}$ were encountered in previous works on vibration control of large structures [27-30], and they were circumvented by using a slightly perturbed state matrix of the form $\hat{\mathbf{A}}=\mathbf{A}-\varepsilon \mathbf{I}$ with a small $\varepsilon>0$. By means of this computational trick, it was possible to overcome the initial feasibility difficulties and to obtain suitable static output-feedback controllers. This approach, however, has proved to be inappropriate for the output-feedback controllers considered in the present paper and, after extensive numerical testing, no satisfactory results have been obtained by using a perturbed state matrix $\hat{\mathbf{A}}$. These facts come to highlight the singular relevance of the matrix $\mathbf{L}$ defined in (14), showing its ability to avoid unfeasibility and to capture the specific properties of the considered control problem.

\section{Numerical results}

In this section, we consider the following control configurations for the quarter-car suspension model:

(i) Controlled system using the active output-feedback controller (43), defined by the two-measurement observed output $\mathbf{y}_{\mathrm{I}}(t)=\left[z_{s}(t)-z_{u}(t), \dot{z}_{s}(t)\right]^{T}$ and the output gain matrix $\mathbf{K}_{\mathrm{I}}$ in (50). This controller will be called output-feedback controller I in the sequel.

(ii) Controlled system using the active output-feedback controller (53), defined by the single-measurement observed output $\mathbf{y}_{\mathrm{II}}(t)=\dot{z}_{s}(t)$ and the output gain $\mathbf{K}_{\mathrm{II}}$ given in (58). For clarity, this controller will be referred to as velocity-feedback controller II in what follows.

(iii) Controlled system using the active state-feedback controller (39), defined by the full state

$$
\mathbf{x}(t)=\left[z_{s}(t)-z_{u}(t), z_{u}(t)-z_{r}(t), \dot{z}_{s}(t), \dot{z}_{u}(t)\right]^{T}
$$

and the state gain matrix $\mathbf{G}_{s}$ in (40).

(iv) Uncontrolled system with no active control implementation.

For these four control configurations, the frequency transfer functions from the road displacement velocity $\dot{z}_{r}(t)$ to the sprung mass acceleration $\ddot{z}_{S}(t)$, to the suspension deflection $z_{s}(t)-z_{u}(t)$, to the tyre deflection $z_{u}(t)-z_{r}(t)$, and to the control force $u(t)$ are presented in Fig.3(a), Fig. 3(b), Fig. 3(c), and Fig. 3(d), respectively. In the graphics, the black dotted line corresponds to the uncontrolled system, the red dash-dotted line corresponds to the outputfeedback controller I, the green solid line pertains to the velocity-feedback controller II, and the blue dashed line represents the state-feedback controlled system, which is taken as a reference to evaluate the performance of the proposed static output-feedback controllers. Looking at the graphics in Fig. 3, it can be clearly appreciated that the frequency response of the output-feedback controller I (which has attained a practically optimal $\gamma$-value) is very similar to the response corresponding to the state-feedback controller. For the velocity-feedback controller II, a small loss of performance with respect to the state-feedback controller can be observed. However, we must recall the severe feedback information constraints imposed on this controller.

To provide a better insight into the behavior exhibited by the proposed output-feedback controllers, we consider the time responses to an isolated bump of the form

$$
z_{r}(t)=\left\{\begin{array}{cc}
\frac{A}{2}\left[1-\cos \left(\frac{2 \pi V}{L} t\right)\right] & \text { if } 0 \leq t \leq \frac{L}{V} \\
0 & \text { otherwise }
\end{array}\right.
$$

where $A$ and $L$ are the bump height and bump length, respectively, and $V$ is the vehicle forward velocity. The following particular values [39]:

$$
A=0.1 \mathrm{~m}, \quad L=5 \mathrm{~m}, \quad V=12.5 \mathrm{~m} / \mathrm{s},
$$

have been taken to conduct the numerical simulations. For the control configurations (i)-(iv), the graphics corresponding to the sprung mass acceleration $\ddot{z}_{S}(t)$ and the control efforts $u(t)$ are respectively presented in Fig. 4(a) and Fig. 4(d), with the same colors and line styles used in the frequency plots. A quick inspection of these figures makes apparent the ability of the active controllers to mitigate the sprung mass acceleration response. It can also be clearly appreciated that the proposed output-feedback controllers achieve similar levels of response mitigation as the 
(a)

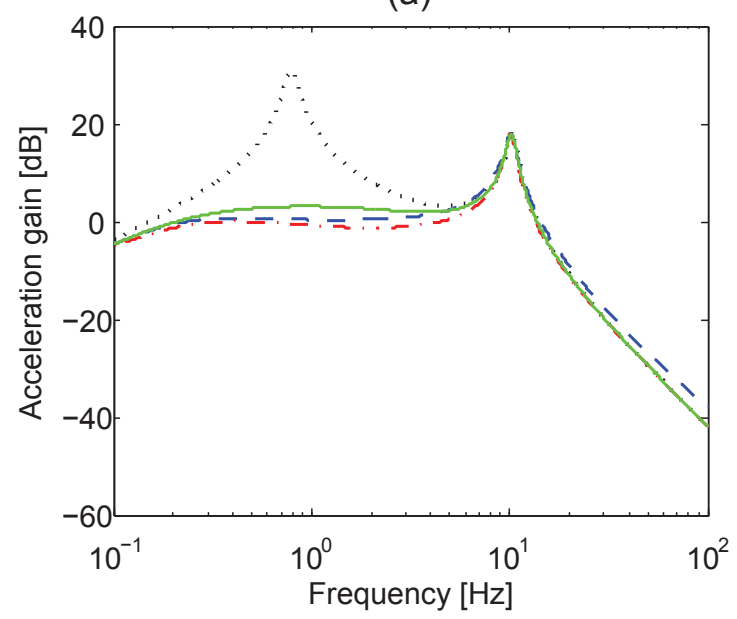

(c)

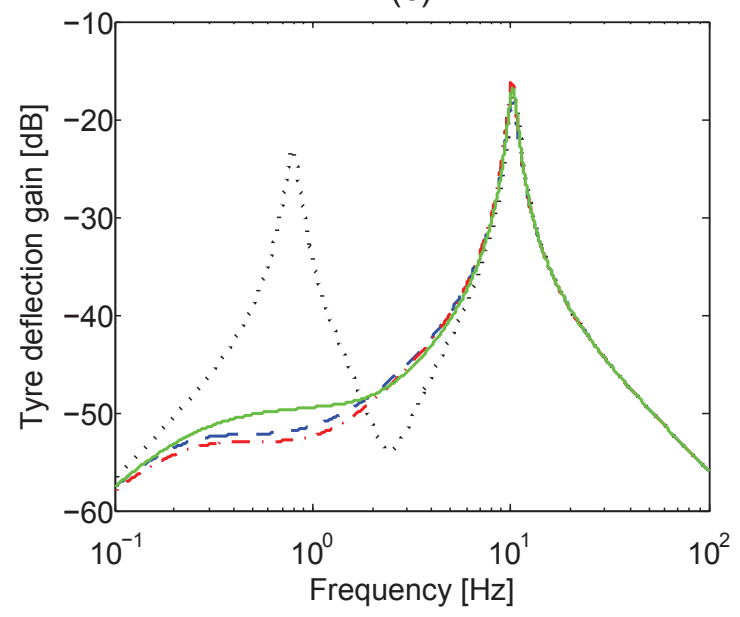

(b)

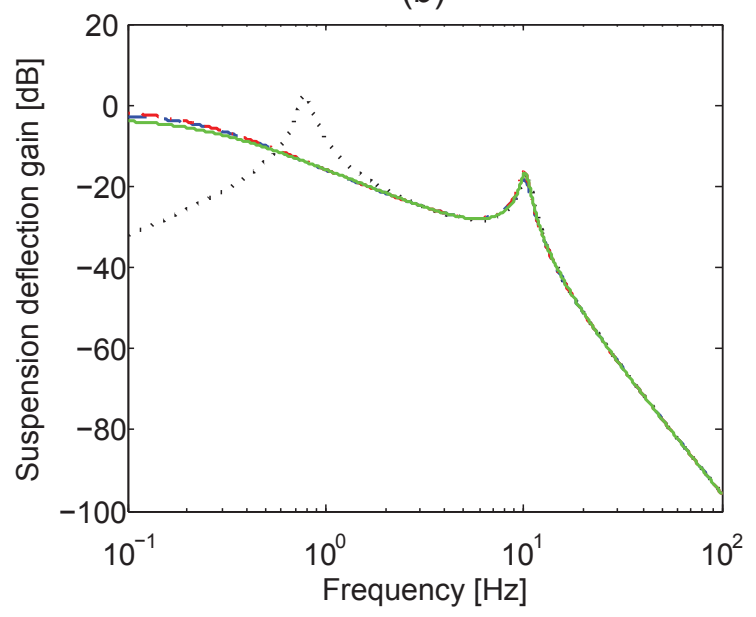

(d)

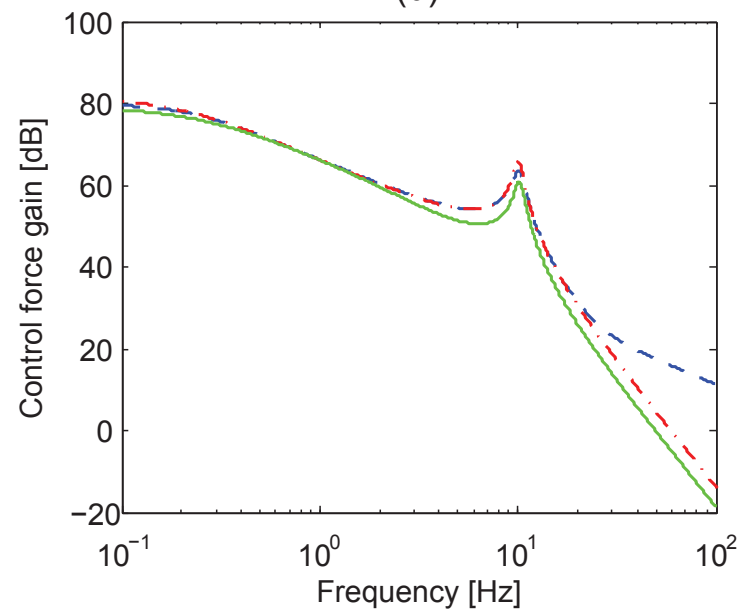

Figure 3: Frequency transfer functions from road displacement velocity to: (a) sprung mass acceleration, (b) suspension deflection, (c) tyre deflection and (d) control effort, corresponding to the output-feedback controller I (red dash-dotted line), velocity-feedback controller II (green solid line), state-feedback (blue dashed line) and uncontrolled (black dotted line) configurations

state-feedback controller, with similar levels of control effort. The graphics in Fig. 4(b) and Fig. 4(c) demonstrate the effectiveness of the active controllers in mitigating the suspension deflection response and the tyre deflection response. In this case, slightly larger peak values are produced by the active controllers during the initial 0.3 seconds when compared with the uncontrolled configuration. These large initial amplitudes, however, are effectively reduced by the active controllers.

Remark 5 As indicated in Remark 2, the state-feedback controller (39) has been intendedly designed to match the behavior of the static output-feedback controller presented in [19], which uses the observed outputs $z_{s}(t)-z_{u}(t), \dot{z}_{s}(t)$ as feedback information and has been computed using a
Genetic Algorithm approach. Consequently, the performances of the static output-feedback controller presented in [19] and the proposed output-feedback controller I are very similar. However, it should be highlighted that the design methodology proposed in Section 2 has made it possible to compute the output-feedback controller I by solving two LMI optimization problems. Moreover, a second output-feedback controller that only uses $\dot{z}_{s}(t)$ as feedback information has also been obtained by solving a single LMI optimization problem. In both cases, after obtaining a satisfactory state-feedback controller, no additional parameter values need to be set to implement the design procedure. 
(a)

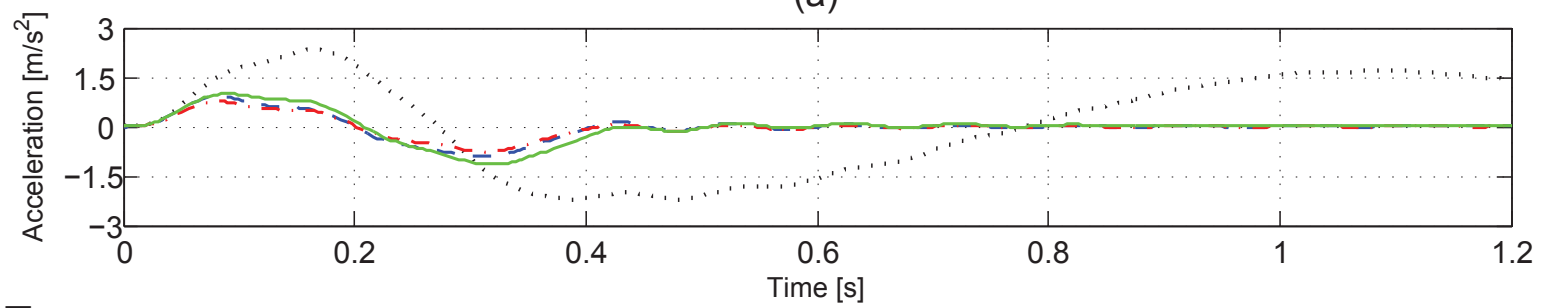

(b)

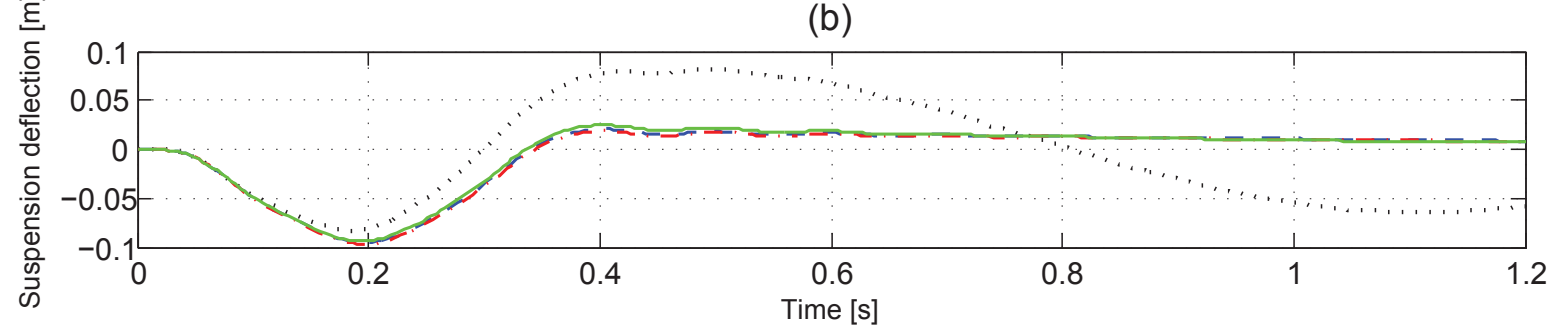

(c)

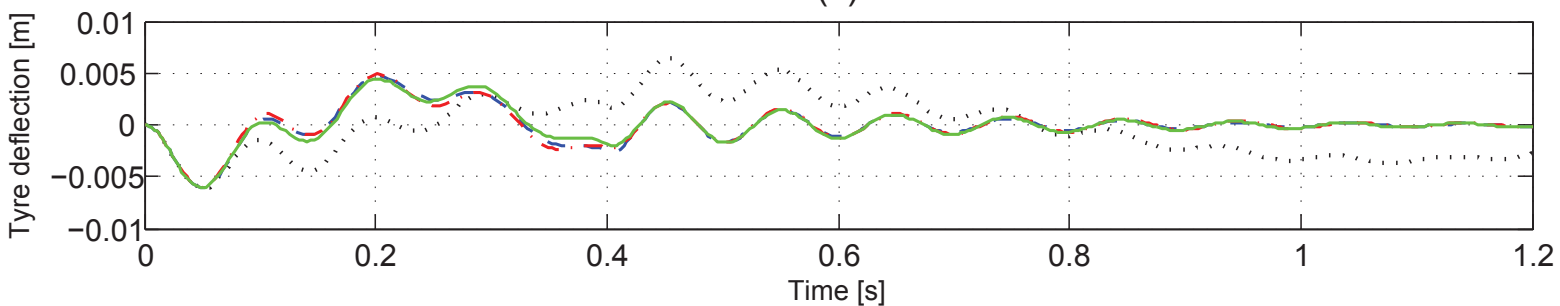

(d)

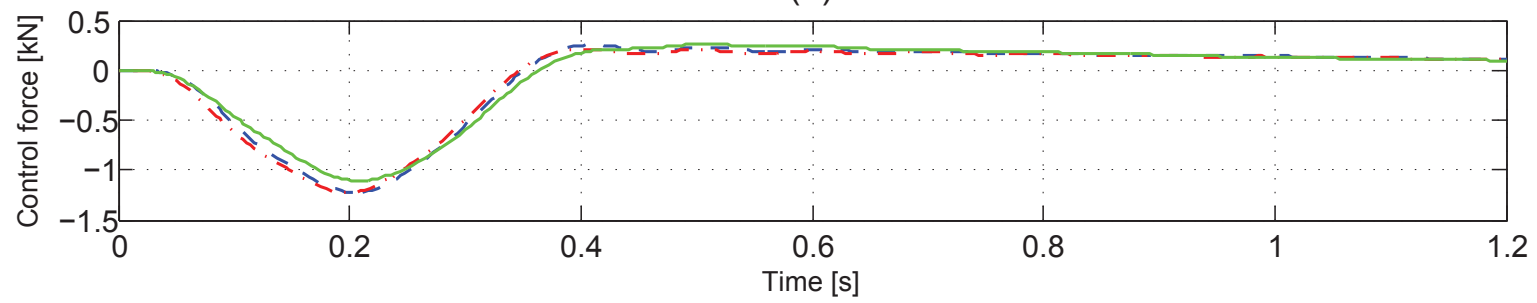

Figure 4: Time response to an isolated bump disturbance: (a) sprung mass acceleration, (b) suspension deflection, (c) tyre deflection and (d) control effort, corresponding to the output-feedback controller I (red dash-dotted line), velocity-feedback controller II (green solid line), state-feedback (blue dashed line) and uncontrolled (black dotted line) configurations

\section{Conclusions and future directions}

In this work, a novel strategy to design static outputfeedback controllers for vehicle suspension systems has been presented. To illustrate the main elements of the new approach, two kinds of static output-feedback $H_{\infty}$ controllers have been designed for a simplified quarter-car suspension system. Numerical simulations show that the proposed static output-feedback $H_{\infty}$ controllers exhibit a good behavior in terms of both frequency and time responses, when compared with the corresponding optimal state-feedback $H_{\infty}$ controller. In fact, from the point of view of $H_{\infty}$ controller design, the values of the $H_{\infty}$-norms show that the proposed static output-feedback controllers are practically optimal. The positive results obtained for this simplified problem clearly indicate that further research effort should be invested in applying the new methodology to more complex scenarios, involving more complete vehicle models such as half-car or full-car models, or more sophisticated control strategies such as non-fragile control or limited frequency designs. A detailed study of discretetime controllers would also be convenient.

\section{Acknowledgments}

This work was supported in part by the Spanish Ministry of Economy and Competitiveness under Grant DPI201232375/FEDER, and by the Norwegian Center of Offshore Wind Energy (NORCOWE) under Grant 193821/S60 from 
the Research Council of Norway (RCN). NORCOWE is a consortium with partners from industry and science, hosted by Christian Michelsen Research. The authors are also grateful to the Editor-in-Chief, the Associate Editor, and the anonymous reviewers, whose valuable comments have contributed to improve the presentation of this paper.

\section{References}

[1] Li, H., Gao, H., Liu., H.: 'Robust quantised control for active suspension systems', IET Control Theory Appl., 2011, 5, (17), pp. 1955-1969.

[2] Sun, W., Li, J., Zhao., Y., Gao, H.: 'Vibration control for active seat suspension systems via dynamic output feedback with limited frequency characteristic', Mechatronics, 2011, 21, (1), pp. 250-260.

[3] Sun, W., Gao, H., Kaynak, O.: 'Finite frequency $H_{\infty}$ control for vehicle active suspension systems', IEEE Trans. Control Syst. Technol., 2011, 19, (2), pp. 416-422.

[4] Prabakar, R.S., Sujatha, C., Narayanan, S.: 'Response of a quarter car model with optimal magnetorheological damper parameters', J. Sound Vib., 2013, 332, (9), pp. 2191-2206.

[5] Sun, W., Gao, H., Kaynak, O.: 'Adaptive backstepping control for active suspension systems with hard constraints', IEEE/ASME Trans. Mechatronics, 2013, 18, (3), pp. 10721079.

[6] Sun, W., Zhao, Z., Gao, H.: 'Saturated adaptive robust control for active suspension systems', IEEE Trans. Ind. Electron., 2013, 60, (9), pp. 3889-3896.

[7] Zhang, Y., Liu, G., Wang, P., Karimi, H.R.: 'Finite frequency vibration control for polytopic active suspensions via dynamic output feedback', Math. Prob. Eng., 2013, 2013, Article ID 598489, 12 p.

[8] Li, H., Jing, X., Karimi, H.R.: 'Output-feedback-based $H_{\infty}$ control for vehicle suspension systems with control delay', IEEE Trans. Ind. Electron., 2014, 61, (1), pp. 436-446.

[9] Zečević, A.I., Šiljak, D.D.: 'Control design with arbitrary information structure constraints', Automatica, 2008, 44, (10), pp. 2642-2647.

[10] Zečević, A.I., Cheng, E., Šiljak, D.D.: 'Control design for large-scale Lur'e systems with arbitrary information structure constraints', Appl. Math. Comput., 2010, 217, (3), pp. $1277-1286$.

[11] Syrmos, V.L., Abdallah, C.T., Dorato, P., Grigoriadis, K.: 'Static output feedback - A survey', Automatica, 1997, 33, (2), pp. 125-137.
[12] Crusius, C.A.R., Trofino, A.: 'Sufficient LMI conditions for output feedback control problems', IEEE Trans. Autom. Control, 1999, 44, (5), pp. 1053-1057.

[13] Prempain, E., Postlethwaite, I.: 'Static output feedback stabilisation with $H_{\infty}$ performance for a class of plants', Syst. Control Lett., 2001, 43, (3), pp. 159-166.

[14] Bara, G.I., Boutayeb, M.: 'Static output feedback stabilization with $H_{\infty}$ performance for linear discrete-time systems', IEEE Trans. Autom. Control, 2005, 50, (2), pp. 250-254.

[15] Feng, J.E., Lam, J., Li, P., Shu, Z.: 'Decay rate constrained stabilization of positive systems using static output feedback', Int. J. Robust Nonlinear Control, 2011, 21, (1), pp. $44-54$.

[16] Blondel, V., Tsitsiklis, J.N.: 'NP-hardness of some linear control design problems', SIAM J. Control Optim., 1997, 35, (6), pp. 2118-2127.

[17] Moerder, D.D., Calise, A.J.: 'Convergence of a numerical algorithm for calculating optimal output feedback gains', IEEE Trans. Autom. Control, 1985, 30, (9), pp. 900-903.

[18] Ghaoui, L.E., Oustry, F., AitRami, M.: 'A cone complementarity linearization algorithm for static output-feedback and related problems', IEEE Trans. Autom. Control, 1997, 42, (8), pp. 1171-1176.

[19] Du, H., Lam, J., Sze, K.Y.: 'Non-fragile output feedback $H_{\infty}$ vehicle suspension control using genetic algorithm', Eng. Appl. Artif. Intel., 2003, 16, (7-8), pp. 667-680.

[20] Gao, H., Lam, J., Wang, C., Wang, Y.: 'Delay-dependent output-feedback stabilisation of discrete-time systems with time-varying state delay', IEE Proc.-Control Theory Appl., 2004, 151, (6), pp. 691-698.

[21] Toscano, R.: 'A simple method to find a robust output feedback controller by random search approach', ISA Trans., 2006, 45, (1), pp. 35-44.

[22] Gadewadikar, J., Lewis, F.L., Xie, L., Kucera, V., AbuKhalaf, M.: 'Parameterization of all stabilizing $H_{\infty}$ static state-feedback gains: application to output-feedback design', Automatica, 2007, 43, (9), pp. 1597-1604.

[23] He, Y., Wu, M., Liu, G.P., She, J.H.: 'Output feedback stabilization for a discrete-time system with a time-varying delay', IEEE Trans. Autom. Control, 2008, 53, (10), pp. 2372-2377.

[24] Shu, Z., Lam, J., Xiong, J.: 'Static output-feedback stabilization of discrete-time markovian jump linear systems: a system augmentation approach', Automatica, 2010, 46, (4), pp. 687-694. 
[25] Du, B., Lam, J., Zou, Y., Shu, Z.: 'Stability and stabilization for markovian jump time-delay systems with partially unknown transition rates', IEEE Trans. Circuits Syst., 2013, 60, (2), pp. 341-351.

[26] Rubió-Massegú, J., Rossell, J.M., Karimi, H.R., PalaciosQuiñonero, F.: 'Static output-feedback control under information structure constraints', Automatica, 2013, 49, (1), pp. 313-316.

[27] Rubió-Massegú, J., Palacios-Quiñonero, F., Rossell, J.M.: 'Decentralized static output-feedback $H_{\infty}$ controller design for buildings under seismic excitation', Earthq. Eng. Struct. Dyn., 2012, 41, (7), pp. 1199-1205.

[28] Palacios-Quiñonero, F., Rubió-Massegú, J., Rossell, J.M., Karimi, H.R.: 'Optimal passive-damping design using a decentralized velocity-feedback $H_{\infty}$ approach', Model. Ident. Control, 2012, 33, (3), pp. 87-97.

[29] Palacios-Quiñonero, F., Rubió-Massegú, J., Rossell, J.M., Karimi, H.R.: 'Discrete-time static output-feedback semidecentralized $H_{\infty}$ controller design: an application to structural vibration control'. Am. Control Conf., 2012, pp. 61266131.

[30] Palacios-Quiñonero, F., Karimi, H.R., Rubió-Massegú, J., Rossell, J.M.: 'Passive-damping design for vibration control of large structures'. Proc. 10th IEEE Int. Conf. Control Autom., ICCA, 2013, pp. 33-38.

[31] Bakka, T., Karimi, H.R.: ' $H_{\infty}$ static output-feedback control design with constrained information for offshore wind turbine system', J. Franklin Inst., 2013, 350, (8), pp. 22442260.

[32] Bakka, T., Karimi, H.R., Christiansen, S.: 'Linear parameter-varying modeling and control of an offshore wind turbine with constrained information', IET Control Theory Appl., 2013, 350, (8), pp. 1-8.

[33] Rubió-Massegú, J., Palacios-Quiñonero, F., Rossell, J.M., Karimi, H.R.: 'Static output-feedback control for vehicle suspensions: a single-step linear matrix inequality approach', Math. Prob. Eng., 2013, 2013, Article ID 907056, $12 \mathrm{p}$.

[34] Palacios-Quiñonero, F., Rubió-Massegú, J., Rossell, J.M., Karimi, H.R.: 'Feasibility issues in static output-feedback controller design with application to structural vibration control', J. Franklin Inst., 2014, 351, (1), pp. 139-155.

[35] Boyd, S., Ghaoui, L.E., Feron, E., Balakrishnan, V.: 'Linear matrix inequalities in system and control theory', SIAM Stud. Appl. Math., (SIAM, Philadelphia, PA, Vol. 15, 1994).
[36] Balas, G.J., Chiang, R.Y., Packard, A.K., Safonov, M.G.: 'Matlab ${ }^{\mathrm{TM}}$ robust control toolbox ${ }^{\mathrm{TM}}$ user's guide: Version 4.2', Natick, MA, USA, 2012.

[37] Tam, P.K.S., Tan, W.: 'A direct composite $H_{\infty}$ controller design for a two-time-scale active suspension system'. Proc. 22nd IEEE Int. Conf. on Ind. Electron., Control, Instrum., IECON, 1996, pp. 1401-1405.

[38] Fialho, I.J., Balas, G.J.: 'Design of nonlinear controllers for active vehicle suspensions using parameter-varying control synthesis', Vehicle Syst. Dyn., 2000, 33, (5), pp. 351-370.

[39] Chen, H., Guo, K.H.: 'Constrained $H_{\infty}$ control of active suspensions: an LMI approach', IEEE Trans. Control Syst. Technol., 2005, 13, (3), pp. 412-421. 